\title{
Inverse Gaussian Modeling of Turbulence-Induced Fading in Free-Space Optical Systems
}

\author{
Nestor D. Chatzidiamantis, Student Member, IEEE, Harilaos G. Sandalidis, \\ George K. Karagiannidis, Senior Member, IEEE, and Michail Matthaiou, Member, IEEE
}

\begin{abstract}
We propose the inverse Gaussian distribution, as a less complex alternative to the classical log-normal model, to describe turbulence-induced fading in free-space optical (FSO) systems operating in weak turbulence conditions and/or in the presence of aperture averaging effects. By conducting goodness of fit tests, we define the range of values of the scintillation index for various multiple-input multiple-output (MIMO) FSO configurations, where the two distributions approximate each other with a certain significance level. Furthermore, the bit error rate performance of two typical MIMO FSO systems is investigated over the new turbulence model; an intensity-modulation/direct detection MIMO FSO system with $Q$-ary pulse position modulation that employs repetition coding at the transmitter and equal gain combining at the receiver, and a heterodyne MIMO FSO system with differential phase-shift keying and maximal ratio combining at the receiver. Finally, numerical results are presented that validate the theoretical analysis and provide useful insights into the implications of the model parameters on the overall system performance.
\end{abstract}

Index Terms-Free-space optical (FSO), inverse gaussian (IG) distribution, log-normal (LN) distribution, performance analysis, turbulence-induced fading.

\section{INTRODUCTION}

F REE-SPACE OPTICAL (FSO) communication systems have recently attracted considerable research interest for a variety of applications. Operating at unlicensed optical wavelengths, FSO systems offer the potential of broadband communication capacity. As the demand for high-speed and tap-proof communications grows, these systems emerge as a cost-effective alternative and/or a complement to radio frequency (RF) counterparts. Additionally, features such as flexibility, rapid deployment time, high security, and robustness to RF interference have rendered FSO systems appealing for disaster recovery and military applications [1].

Despite their significant advantages, there are some major impairments that hamper the widespread deployment of FSO

Manuscript received October 21, 2010; revised March 17, 2011; accepted March 18, 2011. Date of publication March 28, 2011; date of current version May 06, 2011. This paper was presented in part at the IEEE International Conference on Communications (ICC'2010), Cape Town, South Africa, May 2010.

N. D. Chatzidiamantis and G. K. Karagiannidis are with the Department of Electrical and Computer Engineering, Aristotle University of Thessaloniki, Thessaloniki GR-54124, Greece (e-mail: nestoras@auth.gr; geokarag@auth.gr).

H. G. Sandalidis is with the Department of Computer Science and Biomedical Informatics, University of Central Greece, Lamia GR-35100, Greece (e-mail: sandalidis@ucg.gr).

M. Matthaiou is with the Department of Signals and Systems, Chalmers University of Technology, Gothenburg SE-412 96, Sweden (e-mail: michail.matthaiou@chalmers.se).

Digital Object Identifier 10.1109/JLT.2011.2132792 systems. The major performance-limiting factor is their high vulnerability to adverse atmospheric conditions. Rain, fog, and snow are some meteorological phenomena that affect the overall performance. Even in a clear sky, the refractive index of the atmosphere varies, due to inhomogeneities in temperature and pressure, and results in atmospheric turbulence. This causes rapid fluctuations of the received optical signal, known as turbulence-induced fading, that increases error rate, thereby severely affecting the reliability of FSO links [2]

Turbulence-induced fading has been extensively studied in the literature and a number of statistical models have been proposed for describing all different degrees of turbulence severity. One of the most widely used approaches is the log-normal (LN) model, which is considered to be very effective in weak turbulence conditions [2]. Moreover, recent studies and experiments have proved that the LN model is also suitable for describing turbulence when the receiver's aperture is larger than the correlation length of irradiance fluctuations (i.e., when aperture averaging takes place) [3], [4]. Therefore, the LN model has been extensively employed in the performance analysis of FSO systems. Under the assumption of such a model, Zhu and Kahn [5] have studied the error rate performance of FSO links assuming intensity modulation/direct detection (IM/DD) with ON-OFF keying (OOK). In [6], Li and Uysal have evaluated the ergodic capacity for LN turbulence channels. In [7], Navidpour et al. examined the bit error rate (BER) performance of FSO links with spatial diversity employing OOK. The outage probability of FSO systems with multiple transmit/receive apertures has been further evaluated in [8].

Despite its extensive use, the LN distribution is analytically intractable for the BER evaluation of FSO systems. This stems from the fact that the expression for the moment-generating function (MGF) of the LN distribution cannot be derived in closed form. Hence, no analytical expressions are available in the open technical literature for the BER performance analysis over the LN turbulence model; in this case, numerical methods, such as Gauss-Hermite polynomials [7], [9], or Monte Carlo simulations [10], need to be employed. Furthermore, due to the fact that the distribution of the sum of LN variates is not analytically available, the error rate analysis over multiple-input multiple-output (MIMO) FSO systems requires additional approximations [7], [8], whose accuracy deteriorates as the number of the transmit or/and receive apertures increases.

In this paper, we introduce an alternative distribution to the LN distribution, that can efficiently model irradiance fluctuations in weak turbulence conditions or in the presence of aperture averaging, providing simplicity as well. To this end, we propose the inverse Gaussian (IG) distribution. This statistical 
model was originally introduced in [11] as an accurate substitute to the LN distribution for describing shadowing effects in RF wireless communications, while, in the context of optical systems, it has been employed for modeling the statistical behavior of avalanche photo diodes receivers [12].

We first define the range of turbulence conditions, where the IG distribution efficiently approximates the $\mathrm{LN}$ and the sum of LN distributions. Then, we deduce novel analytical expressions for the BER of typical single-input single-output (SISO) and MIMO FSO systems operating in IG turbulence-induced fading. Particularly, two types of typical FSO systems are studied: 1) an IM/DD MIMO FSO system with $Q$-ary pulse position modulation $(Q P P M)$ that employs repetition coding $(\mathrm{RC})$ at the transmitter and equal-gain combining (EGC) at the receiver; and 2) a shot-noise limited heterodyne single-input multiple-output FSO system with differential phase-shift keying (DPSK) that employs maximal-ratio combining (MRC) at the receiver. Numerical results are further provided for both cases to illustrate the accuracy of the proposed approach.

The remainder of this paper is organized as follows. In Section II, we present the IG distribution and introduce its basic statistical parameters. In Section III, the IG model is compared with the LN model and the range of turbulence conditions, where both models converge with a certain level of significance, is defined. In Section IV, the performance of typical FSO systems operating over an IG distributed channel is investigated and concluding remarks are given in Section V.

\section{Modeling Turbulence With the IG Model}

\section{A. SISO FSO Channel}

Let us consider that irradiance fluctuations are modeled by the IG model. The probability density function (PDF) of the IG-distributed irradiance $I$ is given by (see [13, eq. (2.1)])

$$
f_{I}(I)=\sqrt{\frac{\lambda}{2 \pi I^{3}}} \exp \left(-\frac{\lambda(I-\mu)^{2}}{2 \mu^{2} I}\right), \quad I>0
$$

where $\mu>0$ is the parameter related to the mean of the fluctuations and $\lambda>0$ is the scale parameter of the distribution. The $n$th moment of the random variable is given by (see [13, eq. (2.5)])

$$
\mathbb{E}\left[I^{n}\right]=\mu^{n} \sum_{k=0}^{n-1} \frac{(n-1+k) !}{k !(n-1-k) !}\left(\frac{2 \lambda}{\mu}\right)^{-k}
$$

where $\mathbb{E}[\cdot]$ denotes expectation.

Without loss of generality, we can assume that $\mathbb{E}[I]=1$. Using the first moment of (2), it is easily seen that $\mu=1$. Hence, the irradiance scintillation index (SI) $\sigma_{I}^{2}$ is obtained by [2]

$$
\sigma_{I}^{2} \triangleq \frac{\mathbb{E}\left[I^{2}\right]}{(\mathbb{E}[I])^{2}}-1=\frac{1}{\lambda}
$$

and as such (1) can be directly expressed in terms of $\sigma_{I}^{2}$ as

$$
f_{I}(I)=\sqrt{\frac{1}{2 \pi \sigma_{I}^{2} I^{3}}} \exp \left(-\frac{(I-1)^{2}}{2 \sigma_{I}^{2} I}\right) .
$$

Using the derived parameters, the cumulative distribution function (CDF) of the IG model can be written as (see [13, eq. (2.14)])

$$
F_{I}(I)=1-Q\left(\frac{I-1}{\sqrt{I} \sigma_{I}}\right)+\exp \left(\frac{2}{\sigma_{I}^{2}}\right) Q\left(\frac{I+1}{\sqrt{I} \sigma_{I}}\right)
$$

where $Q(\cdot)$ is the Gaussian $Q$-function, defined as $Q(x)=$ $(1) /(\sqrt{2 \pi}) \int_{x}^{\infty} \exp \left(-\left(t^{2}\right) /(2)\right) d t$.

Finally, the MGF, defined as

$$
M_{I}(s) \triangleq \int_{0}^{\infty} \exp (-s x) f_{I}(x) d x,
$$

can be deduced in closed form as (see [13, eq. (2.4)])

$$
M_{I}(s)=\exp \left(\frac{1}{\sigma_{I}^{2}}\left(1-\sqrt{1+2 \sigma_{I}^{2} s}\right)\right) .
$$

\section{B. MIMO FSO Channel}

In the case of MIMO FSO systems, the distribution of the sum of multiple IG-distributed random variables (RVs) is needed. Let us define as $S$ the sum of $L$ independent identically distributed (i.i.d.) IG RVs, i.e.,

$$
S=\sum_{l=1}^{L} I_{l}
$$

where $I_{l}$ is IG distributed with PDF given by (4). According to [13, p. 13], $S$ will also be IG distributed with PDF given by

$$
f_{S}(I)=\sqrt{\frac{L^{2}}{2 \pi \sigma_{I}^{2} I^{3}}} \exp \left(-\frac{(I-L)^{2}}{2 \sigma_{I}^{2} I}\right)
$$

and $\mathrm{CDF}$ given by

$$
F_{S}(I)=1-Q\left(\frac{I-L}{\sqrt{I} \sigma_{I}} L\right)+\exp \left(\frac{2 L^{2}}{\sigma_{I}^{2}}\right) Q\left(\frac{I+L}{\sqrt{I} \sigma_{I}} L\right) .
$$

\section{COMPARISON With THE LN MODEL}

In this section, we demostrate that the IG distribution can serve as a more tractable and analytically friendlier alternative to the LN distribution, under weak turbulence conditions. Specifically, we employ Kolmogorov-Smirnov (KS) goodness of fit statistical tests in order to measure the difference between the two models. These tests measure the maximum value of the absolute difference between the CDFs of two distributions (see [14, pp. 272-273]), respectively. Hence, for the case under consideration, the KS test statistic is defined as

$$
T \triangleq \max \left|F_{S}(x)-F_{y}(x)\right|
$$

where $F_{S}(\cdot)$ is the CDF of the sum of $L$ i.i.d. IG RVs analytically evaluated by (9) and $F_{y}(\cdot)$ is the CDF of the sum of $L$ i.i.d. LN RVs. It should be noted that in the case of $L=1, F_{y}(\cdot)$ is given by [15]

$$
F_{y}(x)=1-Q\left(\frac{\ln \left(x \sqrt{\sigma_{I}^{2}+1}\right)}{\sqrt{\ln \left(\sigma_{I}^{2}+1\right)}}\right) .
$$

However, there is no closed-form expression when $L>1$. 


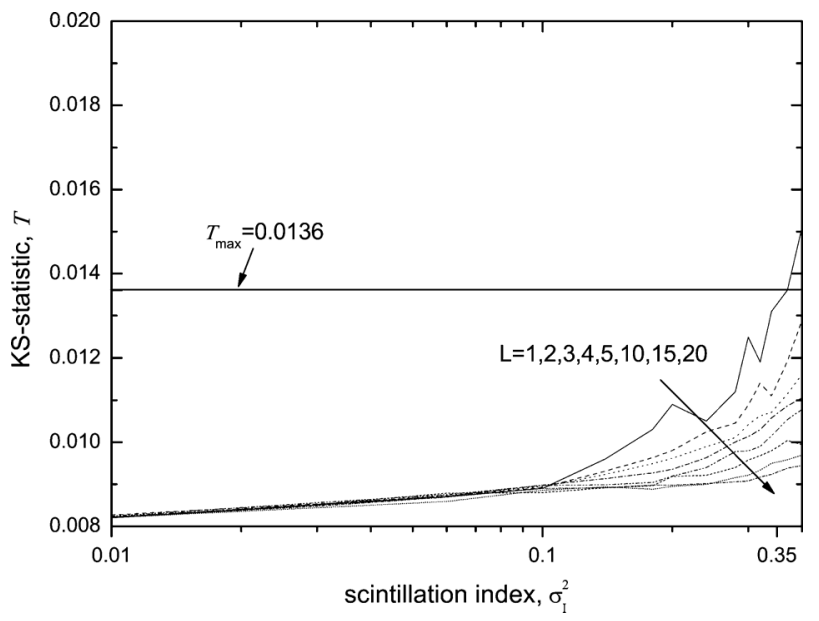

Fig. 1. Hypothesis testing distribution using the KS goodness-of-fit test for the IG to approximate the LN distribution with a 5\% significance level.

Definition 1: We define $\boldsymbol{H}_{0}$ as the null hypothesis under which the IG-distributed data belongs to the CDF of the LN distribution $F_{y}(\cdot)$.

To test $\boldsymbol{H}_{0}$, the KS goodness-of-fit test compares the test statistic $T$ to a critical level $T_{\max }$ for a given significance level $\alpha$. Any hypothesis for which $T>T_{\max }$ is rejected with significance $1-\alpha$, while any hypothesis for which $T<T_{\max }$ is accepted with the same level of significance.

Fig. 1 depicts the KS test statistic for different values of $L$ and $\sigma_{I}^{2}$, which corresponds to various MIMO FSO configurations and different degrees of turbulence severity, respectively. The presented test results have been obtained by averaging the results of 60 simulation runs, each for at least $10^{4}$ samples of IG-distributed data. The critical value $T_{\max }$, used for comparison reasons, is equal to $T_{\max }=0.0136$ calculated by [14, eqs (9)-(73)] when a significance level of $\alpha=5 \%$ is considered. It is clearly illustrated that for a SISO link $(L=1)$, the hypothesis $\boldsymbol{H}_{0}$ is accepted with $95 \%$ significance until $\sigma_{I}^{2}$ reaches the value of 0.35 , which corresponds to the weak turbulence regime. Furthermore, when increasing $L$, it is observed that the $\mathrm{KS}$ statistic is reduced, indicating that the accuracy of the IG model improves as the number of the transmit and/or receive apertures increases.

\section{Performance Analysis Over IG Turbulence Fading}

In this section, we present a detailed performance analysis of typical MIMO FSO systems operating over weak turbulence conditions or in the presence of aperture averaging effects, by considering the IG distribution as the model for describing turbulence-induced fading.

\section{A. QPPM IM/DD System}

We consider an IM/DD MIMO FSO system of $M$ transmit and $N$ receive apertures that employs $Q P P M$. For $Q P P M$, the symbol period $T_{S}$ is divided into $Q$ equal nonoverlapping time slots of duration $T_{p}=T_{S} / Q$ and a digital message, comprised of $\log _{2} Q$ bits, is sent by pulsing the laser's intensity in one of these slots. Furthermore, RC is applied at the transmitter, and therefore, the same optical signal is transmitted through every aperture.

At the receiver, the Poisson photon-counting detection process is assumed [10] and the received signal is a vector of $Q$ Poisson observations that correspond to the photon counts at each time slot. The average number of photoelectrons per slot when a pulse is transmitted is denoted by $n_{s}$ and is given by

$$
n_{s}=\frac{\eta P_{r} T_{p}}{h \nu}
$$

where $\eta$ represents the photodetector's optical-to-electrical efficiency, $h$ is Planck's constant in Joules per Hertz, $\nu$ is the frequency of the optical signal in Hertz, and $P_{r}$ is the total power incident on the array of the photodetectors at the time slot. Similarly, the average number of photoelectrons per slot and per receive aperture due to background noise $n_{b}$ is defined as

$$
n_{b}=\frac{\eta P_{b} T_{p}}{h \nu}
$$

where $P_{b}$ is the power incident on the photodetector due to background noise. Hence, the probability mass function (PMF) of the received signal at the $n$th receive photodetector is given by (14), shown at the bottom of the page.

In the earlier equation, $s_{q} \in\{0,1\}$ depending on whether a pulse is transmitted in time slot $q$, while $I_{m n}$ is the fading coefficient between the $m$ th transmit and $n$th receive apertures, which remains constant during the symbol period.

Furthermore, we consider EGC as the combining method of the received signals from each aperture, since it offers performance close to the optimum combining method without monitoring the channel gains [10]. Hence, the combined signal can be expressed as

$$
\boldsymbol{r}=\sum_{n=1}^{N} \boldsymbol{r}_{n}
$$

while its PMF is equal to

$$
\begin{array}{r}
\operatorname{Pr}\left\{\boldsymbol{r} \mid \boldsymbol{s}, I_{S}\right\}=\prod_{q=1}^{Q} \frac{\left(\frac{n_{s}}{M} s_{q} I_{S}+N n_{b}\right)^{\sum_{n=1}^{N} r_{q n}}}{\left(\sum_{n=1}^{N} r_{q n}\right) !} \\
\quad \times \exp \left(-\frac{n_{s}}{M} s_{q} I_{S}-N n_{b}\right)
\end{array}
$$

${ }^{1}$ Taking into consideration the coherence time of the FSO channel and the signaling rates of interest, this assumption is quite realistic according to [16].

$$
\operatorname{Pr}\left\{\boldsymbol{r}_{n} \mid \boldsymbol{s}, I\right\}=\prod_{q=1}^{Q} \operatorname{Pr}\left\{r_{q n} \mid s_{q}, I\right\}=\prod_{q=1}^{Q} \frac{\left(\frac{n_{s} s_{q}}{M} \sum_{m=1}^{M} I_{m n}+n_{b}\right)^{r_{q n}} \exp \left(-\frac{n_{s} s_{q}}{M} \sum_{m=1}^{M} I_{m n}-n_{b}\right)}{r_{q n} !}
$$


where

$$
I_{S}=\sum_{m=1}^{M} \sum_{n=1}^{N} I_{m n} .
$$

1) Average BER Performance: The conditioned on $I_{S}$ BER can be computed by combining [10, eq. (14)] with [17, eq. (3)]

$$
\begin{aligned}
& \text { as } \\
& \begin{aligned}
& P_{b}\left(e \mid I_{s}\right)=\frac{1}{2(Q-1)} \\
& \times \sum_{k=0}^{\infty} \frac{p_{1}(k)}{p_{0}(k)}\left[p_{0}(k) Q-P_{o}(k)^{Q}+P_{o}(k-1)^{Q}\right]
\end{aligned}
\end{aligned}
$$

where

$$
\begin{aligned}
& p_{1}(i)=\frac{\left(\frac{n_{s}}{M} I_{S}+N n_{b}\right)^{i} \exp \left(-\frac{n_{s}}{M} I_{S}-N n_{b}\right)}{i !} \\
& p_{0}(i)=\frac{\left(N n_{b}\right)^{i} e^{-N n_{b}}}{i !}
\end{aligned}
$$

and

$$
P_{0}(k)=\sum_{i=0}^{k} p_{0}(i)=\frac{\Gamma\left(k+1, N n_{b}\right)}{\Gamma(k+1)} .
$$

In the earlier equations, $\Gamma(\cdot)$ and $\Gamma(\cdot, \cdot)$ denote the Gamma (see [18, eq. (8.310)]) and the upper incomplete Gamma (see $[18$, eq. (8.352.2)]) functions, respectively. Hence, the average BER can be obtained by

$$
\begin{aligned}
P_{b}(e)= & \frac{1}{2(Q-1)} \\
& \times \sum_{k=0}^{\infty}\left[p_{0}(k) Q-P_{o}(k)^{Q}+P_{o}(k-1)^{Q}\right] g(k)
\end{aligned}
$$

where

$$
g(k)=\int_{0}^{\infty}\left(\frac{n_{s}}{M N n_{b}} I_{S}+1\right)^{k} \exp \left(-\frac{n_{s}}{M} I_{S}\right) f_{I_{S}}\left(I_{S}\right) d I_{S}
$$

When the IG turbulence-induced fading model is considered, (23) can be rewritten, according to (8), as

$$
\begin{aligned}
g_{\mathrm{IG}}(k)=M N & \sqrt{\frac{1}{2 \pi \sigma_{I}^{2}}} \int_{0}^{\infty} I_{s}^{-\frac{3}{2}}\left(\frac{n_{s}}{M N n_{b}} I_{s}+1\right)^{k} \\
& \times \exp \left(-\frac{n_{s}}{M} I_{s}-\frac{\left(I_{s}-M N\right)^{2}}{2 \sigma_{I}^{2} I_{S}}\right) d I_{s} .
\end{aligned}
$$

The earlier equation contains an integral of the form

$$
\int_{0}^{\infty} x^{-r}(a x+1)^{k} \exp \left(-b x-\frac{c}{x}\right) d x
$$

which can be solved in closed form as in the Appendix. Hence, (24) can be evaluated by

$$
\begin{aligned}
& g_{\mathrm{IG}}(k)=2 \sqrt{\frac{M N}{2 \pi \sigma_{I}^{2}}} \exp \left(\frac{M N}{\sigma_{I}^{2}}\right) \sum_{j=0}^{k}\left(\begin{array}{c}
k \\
j
\end{array}\right)\left(\frac{n_{s}}{n_{b}}\right)^{j} \\
& \times\left(1+2 \frac{n_{s}}{M} \sigma_{I}^{2}\right)^{\frac{1}{4}-\frac{j}{2}} K_{j-\frac{1}{2}}\left(\frac{M N \sqrt{1+2 \frac{n_{s}}{M} \sigma_{I}^{2}}}{\sigma_{I}^{2}}\right)
\end{aligned}
$$

TABLE I

Required Values of $K$ to Achieve a Relative Truncation ERROR OF LESS THAN $10^{-9}$ IN (22)

\begin{tabular}{|c||c|c|c||c|c|}
\cline { 3 - 6 } \multicolumn{1}{c||}{} & \multicolumn{2}{c||}{$M=1, N=1$} & \multicolumn{2}{c|}{$M=2, N=2$} \\
\hline \hline$E_{b}$ & 0.01 & 0.1 & 0.35 & 0.1 & 0.35 \\
\hline \hline-190 & 67 & 67 & 67 & 117 & 116 \\
\hline-185 & 67 & 67 & 67 & 117 & 117 \\
\hline-180 & 69 & 69 & 70 & 121 & 121 \\
\hline-175 & 75 & 75 & 77 & 131 & 131 \\
\hline-170 & 88 & 85 & 82 & 150 & 145 \\
\hline
\end{tabular}

which can be rewritten, using [18, eq. (8.468)], as

$$
\begin{aligned}
g_{\mathrm{IG}}(k)= & \exp \left(\frac{M N}{\sigma_{I}^{2}}\left(1-\sqrt{1+2 \frac{n_{s}}{M} \sigma_{I}^{2}}\right)\right) \\
& \times\left(1+\sum_{j=1}^{k} \sum_{h=0}^{j-1}\left(\begin{array}{c}
k \\
j
\end{array}\right)\left(1+2 \frac{n_{s}}{M} \sigma_{I}^{2}\right)^{-\frac{j+h}{2}}\right. \\
& \left.\times\left(\frac{n_{s}}{n_{b}}\right)^{j} \frac{(j-1+h) !}{h !(j-1-h) !\left(\frac{2 M N}{\sigma_{I}^{2}}\right)^{h}}\right) .
\end{aligned}
$$

Hence, the average BER can be analytically evaluated by combining (22) with (26) or (27). It should be noted that no closed-form solution is available for (23) when LN turbulence model is considered. In that case, the approximation for the sum of $\mathrm{LN}$ variates [8], in conjunction with Gauss-Hermite integration methods, needs to be employed [9].

In the case of $n_{b}=0$ (quantum-limited reception), the average BER is obtained by (see [10, eq. (18)])

$$
P_{b}(e)=\frac{1}{2} \int_{0}^{\infty} \exp \left(-\frac{n_{s}}{M} I_{S}\right) f_{I_{s}}\left(I_{S}\right) d I_{s} .
$$

Thus, for IG turbulence-induced fading, (28) can be analytically evaluated, using (6), as

$$
P_{b}(e)=\frac{1}{2} \exp \left(\frac{M N}{\sigma_{I}^{2}}\left(1-\sqrt{1+2 \frac{n_{s}}{M} \sigma_{I}^{2}}\right)\right) .
$$

In practice, a finite number of terms $K$ will be used in the evaluation of (22) and we denote the resulting BER approximation by $P_{b}(e, K)$. Table I provides the required $K$ to achieve a relative approximation error $\left|P_{b}(e)-P_{b}(e, K)\right| / P_{b}(e)$ of less than $10^{-9}$, when $M=1, N=1$ or $M=2, N=2, Q=2$, and for different values of $\sigma_{I}^{2}$ and energy per bit, while $P_{b}(e)$ is calculated with $K=300$ (which is confirmed by simulations).

2) Numerical Results: In the following, we present numerical results for the BER performance of the $Q P P M$ MIMO FSO system under consideration for various deployments of transmit and/or receive apertures and for different values of SI. Adopting previously reported parameters [10], we set the photodetector's optical-to-electrical efficiency $\eta=0.5$, the optical carrier's frequency $\nu=1.94 \times 10^{14} \mathrm{~Hz}$, and $E_{\text {back }}=P_{b} T_{p}=-170 \mathrm{dBJ}$ which corresponds to $n_{b}=39$. Furthermore, all of the presented results are plotted in terms of transmitted energy per bit, defined as $E_{b}=P_{r} T_{p} / \log _{2} Q$.

Fig. 2 illustrates the average BER performance of a SISO $Q P P M$ FSO system operating in weak turbulence conditions. Specifically, the BER of the IG model, derived by (26) and (22), 


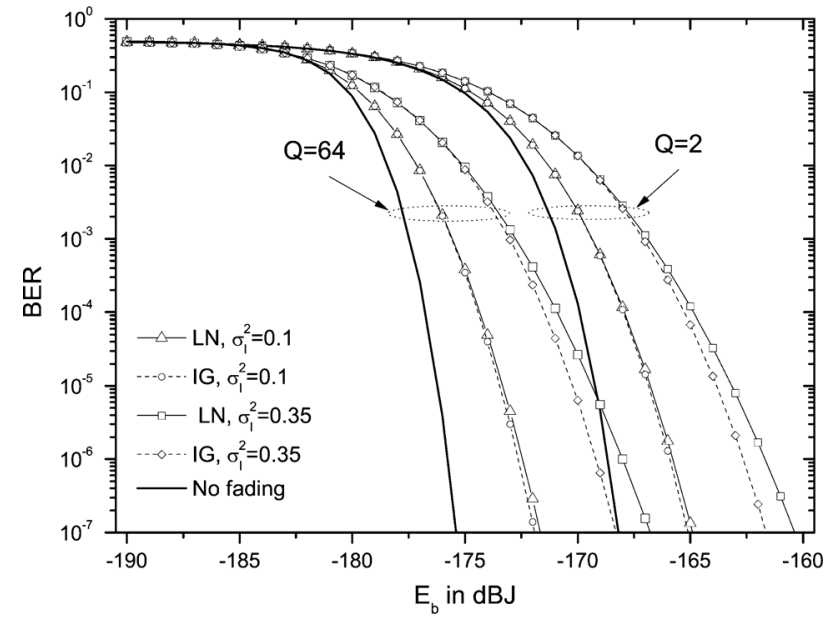

Fig. 2. BER performance of $Q P P M$ systems for different SIs and modulation orders, assuming background energy $E_{\mathrm{back}}=-170 \mathrm{~dB} \cdot \mathrm{J}$ and $M=N=1$.

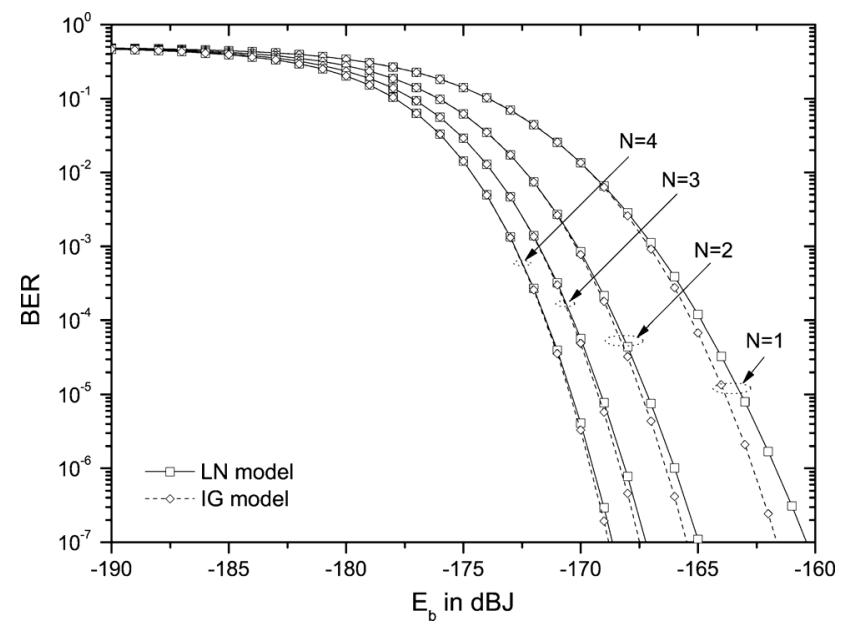

Fig. 3. BER performance of MIMO $Q P P M$ systems for different values of $N$, assuming $M=1$, background energy $E_{\text {back }}=-170 \mathrm{~dB} \cdot \mathrm{J}, Q=2$ and SI 0.35 .

is depicted for different values of $\sigma_{I}^{2}$ and modulation orders $Q$. Further, BER curves, assuming either the LN turbulence model or the absence of turbulence, are also provided for comparison. As it is clearly illustrated, the analytical results using the IG model are very close to the performance of the LN model, derived by numerical integrations. Specifically, they lie within 2 $\mathrm{dB} \cdot \mathrm{J}$ for practical values of BER in all turbulence conditions considered $\left(\sigma_{I}^{2}\right.$ less than 0.35$)$. Moreover, it can be easily observed that the performance of the IG model acts as a tight lower bound, whose tightness looses as the $\sigma_{I}^{2}$ increases, irrespective of the assumed modulation order.

Furthermore, Fig. 3 depicts the BER performance of the $Q P P M$ FSO system for different MIMO FSO systems, assuming $Q=2$ and $\sigma_{I}^{2}=0.35$. The performance over the IG turbulence model comes closer to the performance over the LN model, as the number of the receive (and/or transmit) apertures increases. Thus, it can be concluded that the accuracy of the proposed analytical expressions improves for larger MIMO FSO configurations. In contrast, the numerical methods used when the LN model is considered tend to become cumbersome and more computationally heavy.

\section{B. Heterodyne DPSK System}

In order to obtain better insights into the performance analysis of MIMO FSO systems over the IG model, we now consider a coherent shot-noise limited DPSK system. Henceforth, it is assumed that the diameters of the receivers' apertures are smaller than the characteristic length of the wavefront's phase perturbations. Hence, fades induced by wavefront distortions are ignored [19].

1) Average BER Performance: This type of FSO systems is shot-noise limited, and therefore, the conditioned on the fading intensity $I$ BER can be obtained by [20]

$$
P(e \mid I)=\frac{1}{2} \exp (-\gamma)
$$

where

$$
\begin{aligned}
\gamma= & \bar{\gamma} I \\
& \text { and } \\
\bar{\gamma}= & \frac{\eta A T_{s}}{h \nu}
\end{aligned}
$$

denote the instantaneous and average SNR, respectively. In the earlier equation, $A$ represents the detector area in square meters and $T_{s}$ the DPSK symbol duration in seconds.

Following the same assumptions as in [20], we consider that irradiance remains constant over a symbol's interval. That is, we assume that the duration of $T_{s}$ is smaller than the coherence time of the channel, thus having a constant amplitude scenario for the received signal [21]. Hence, for the slow-fading environment under consideration, the average BER $P(e)$ can be obtained by averaging (30) over the PDF of $I$ [22], i.e.,

$$
P(e)=\int_{0}^{\infty} P(e \mid I) f_{I}(I) d I .
$$

Using (6), the average BER when the IG turbulence-induced fading model is considered can be derived as

$$
P(e)=\frac{1}{2} \exp \left(\frac{1}{\sigma_{I}^{2}}\left(1-\sqrt{1+2 \sigma_{I}^{2} \bar{\gamma}}\right)\right) .
$$

Note that there is no closed-form expression for the MGF of the LN distribution. Therefore, in the case of LN turbulence fading, the average BER is obtained in terms of Gauss-Hermite polynomials (see [23, eq. (2.54)]).

Furthermore, when reception by $L$ receive apertures is considered, the instantaneous SNR at the output of a receiver that applies MRC is given by

$$
\gamma_{L}=\sum_{i=1}^{L} \gamma_{i}
$$

which for the case of equal average SNR per receive aperture can be written as

$$
\gamma_{L}=\bar{\gamma} \sum_{i=1}^{L} I_{i}
$$




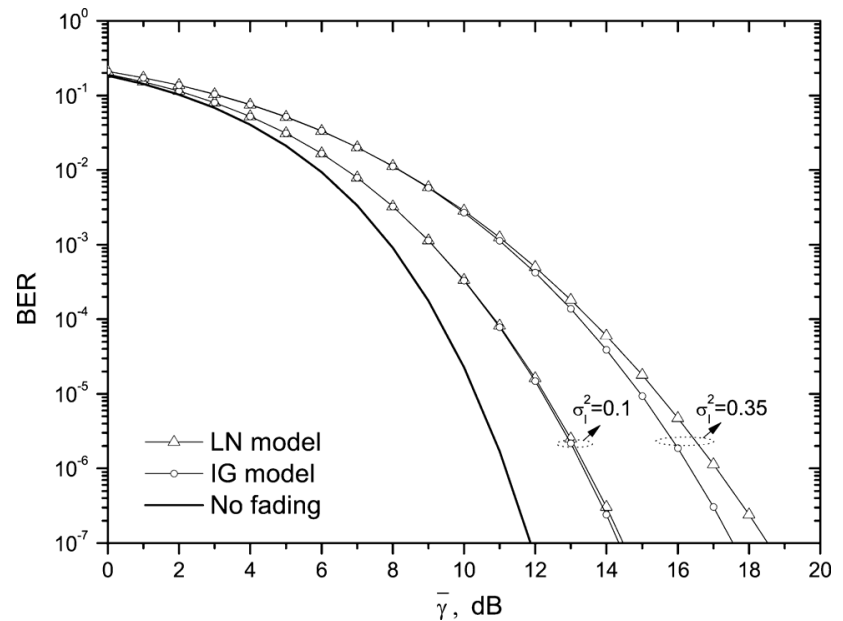

Fig. 4. BER performance of DPSK FSO systems for different SIs and $L=1$.

Hence, under the assumption of independent fading among the diversity branches, ${ }^{2}$ the average BER of the multibranch DPSK FSO system becomes

$$
P(e)=\frac{1}{2} \exp \left(\frac{L}{\sigma_{I}^{2}}\left(1-\sqrt{1+2 \sigma_{I}^{2} \bar{\gamma}}\right)\right) .
$$

2) Numerical Results: Fig. 4 illustrates the average BER performance of a heterodyne SISO DPSK FSO system assuming weak turbulence conditions. Analytical results, obtained by (34), are plotted in comparison with the performance of the LN turbulence model for $\sigma_{I}^{2}$ less than 0.35. A BER curve without turbulence is provided as well. As it is clearly depicted, the performance over the IG turbulence model is very close to the performance over the LN model. Specifically, it lies within 2 $\mathrm{dB}$ for the BER values of interest. Once again, it is observed that the performance of SISO coherent DPSK systems in IG turbulence fading acts as a tight lower bound; the lower the SI the tighter the bound.

Furthermore, Fig. 5 illustrates the BER performance of a DPSK FSO system with multiple receive apertures and employing MRC, assuming $\sigma_{I}^{2}=0.35$.

Clearly, by increasing the number of diversity branches, the accuracy of the proposed distribution improves.

\section{CONCLUSION}

We proposed the IG distribution, as a less complex alternative to LN, to describe turbulence-induced fading in FSO systems operating in weak turbulence conditions and/or in the presence of aperture averaging. By conducting goodness of fit tests, we defined the range of values of the SI, where the two distributions approximate each other, with a certain significance level and for different MIMO FSO deployments. Specifically, it was observed that the IG model efficiently approximates the LN model when $\sigma_{I}^{2}$ is less than 0.35 , while its accuracy increases as the number of transmit and/or receive apertures improves. As part of our performance analysis, we derived novel analytical expressions for the BER of two typical MIMO FSO systems: an

\footnotetext{
${ }^{2} \mathrm{An}$ assumption which is realistic by placing the transmit and receive apertures just a few centimeters away [8].
}

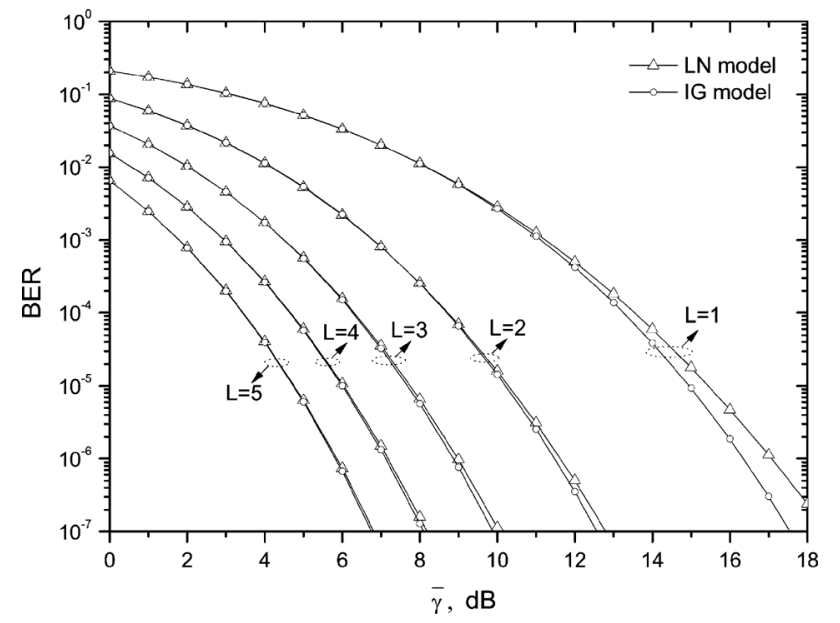

Fig. 5. BER performance of DPSK FSO systems for SI 0.35 and different values of $L$.

IM/DD FSO system with $Q P P M$ and a heterodyne FSO system with DPSK. Numerical examples were provided to demonstrate the accuracy of the proposed approach in the turbulence regime under consideration.

\section{APPENDIX}

This Appendix provides an analytical solution for the integral of the form

$$
f(k)=\int_{0}^{\infty} x^{-r}(a x+1)^{k} \exp \left(-\left(b x+\frac{c}{x}\right)\right) d x
$$

where the variable $k$ is a positive integer. To the best of the authors' knowledge, there is no closed-form solution for the above integral. Taking into consideration that $k \in \mathbb{Z}^{+}$, the term $(a x+1)^{k}$ can be rewritten, according to the binomial theorem (see $[18$, eq. $(1.111)])$, as

$$
(a x+1)^{k}=\sum_{j=0}^{k}\left(\begin{array}{l}
k \\
j
\end{array}\right)(a x)^{j}
$$

and hence (A.1) is rewritten as

$$
f(k)=\sum_{j=0}^{k}\left(\begin{array}{l}
k \\
j
\end{array}\right) a^{j} \int_{0}^{\infty} x^{j-r+1-1} \exp \left(-\left(b x+\frac{c}{x}\right)\right) d x .
$$

Using [18, eq. (3.471.9)], a closed-form solution is obtained as

$$
f(k)=2 \sum_{j=0}^{k}\left(\begin{array}{l}
k \\
j
\end{array}\right) a^{j}\left(\frac{c}{b}\right)^{\frac{1-r+j}{2}} K_{1-r+j}(2 \sqrt{b c})
$$

where $K_{\nu}(\cdot)$ is the $\nu$ th-order modified Bessel function of the second kind (see [18, eq. (8.432.9)]). This concludes the proof.

\section{REFERENCES}

[1] H. Willebrand and B. S. Ghuman, Free Space Optics: Enabling Optical Connectivity in Todays Networks. Indianapolis, IN: Sams, 2002. 
[2] L. Andrews, R. L. Philips, and C. Y. Hopen, Laser Beam Scintillation With Applications. Bellingham, WA: SPIE, 2001.

[3] F. S. Vetelino, C. Young, L. Andrews, and J. Recolons, "Aperture averaging effects on the probability density of irradiance fluctuations in moderate-to strong turbulence," Appl. Opt., vol. 46, no. 11, pp. 2099-2108, Apr. 2007.

[4] N. Perlot and D. Fritzsche, "Aperture-averaging- theory and measurements," Proc. SPIE-Int. Soc. Opt. Eng., vol. 5338, pp. 233-242, Jul. 2004.

[5] X. Zhu and J. M. Kahn, "Free-space optical communication through atmospheric turbulence channels," IEEE Trans. Commun., vol. 50, no. 8, pp. 1293-1300, Aug. 2002.

[6] J. T. Li and M. Uysal, "Optical wireless communications: System model, capacity and coding," in Proc. IEEE Veh. Tech. Conf., Orlando, FL, Oct. 2003, pp. 168-172.

[7] S. M. Navidpour, M. Uysal, and M. Kavehrad, "BER perfomance of freespace optical transmission with spatial diversity," IEEE Trans. Wireless Commun., vol. 6, no. 8, pp. 2813-2819, Aug. 2007.

[8] E. Lee and V. Chan, "Part 1: Optical communication over the clear turbulent atmospheric channel using diversity," IEEE J. Sel. Areas Commun., vol. 22, no. 9, pp. 1896-1906, Nov. 2004.

[9] W. Gappmair and S. S. Muhammad, "Error performance of terrestrial FSO links modelled as PPM/Poisson channels in turbulent atmosphere," IET Electron. Lett., vol. 43, no. 5, pp. 63-64, Mar. 2007.

[10] S. G. Wilson, M. Brandt-Pearce, Q. Qao, and J. H. Leveque, "Free-space optical MIMO transmission with Q-ary PPM," IEEE Trans. Commun., vol. 53, no. 8, pp. 1402-1411, Aug. 2005.

[11] Karmeshu and R. Agrawal, "On efficacy of Rayleigh-inverse Gaussian distribution over K-distribution for wireless fading channels," Wireless Commun. Mobile Comput., vol. 7, no. 1, pp. 1-7, Jan. 2007.

[12] K. R. Baker, "On the WMC density as an inverse Gaussian probability density," IEEE Trans. Commun., vol. 44, no. 1, pp. 15-17, Jan. 1996.

[13] R. S. Chhikara and J. L. Folks, The Inverse Gaussian Distribution: Theory, Methodology, and Applications. New York: Marcel Dekker, 1989.

[14] A. Papoulis, Probability, Random Variables, and Stochastic Processes, 3rd ed. New York: McGraw-Hill, 1991.

[15] D. Giggenbach and H. Henniger, "Fading-loss assessment in atmospheric free-space optical communication links with on-off keying," Opt. Eng., vol. 47, no. 4, p. 046001, Apr. 2008.

[16] D. J. T. Heatley, D. R. Wisely, I. Neild, and P. Cochrane, "Optical wireless: The story so far," IEEE Commun. Mag., vol. 36, no. 2, pp 72-74, Dec. 1998.

[17] J. Hamkins, "Accurate computation of the perfromance of M-ary orthogonal signalling on a discrete memoryless channel," IEEE Trans. Commun., vol. 52, no. 11, pp. 1844-1845, Nov. 2004.

[18] I. S. Gradshteyn and I. M. Ryzhik, Table of Integrals, Series, and Products, 7th ed. New York: Academic, 2007.

[19] N. Perlot, "Turbulence-induced fading probability in coherent optical communication through the atmosphere," Appl. Opt., vol. 46, no. 29 pp. 7218-7226, Oct. 2007.

[20] K. Kiasaleh, "Performance of coherent DPSK free-space optical communication systems in K-distributed turbulence," IEEE Trans. Commun., vol. 54, no. 4, pp. 604-607, Apr. 2006.

[21] H. G. Sandalidis, T. A. Tsiftsis, and G. K. Karagiannidis, "Optical wireless communications with heterodyne detection over turbulence channels with pointing errors," J. Lightw. Technol., vol. 27, no. 12, pp. $4440-4445$, Oct. 2009.

[22] T. A. Tsiftsis, "Performance of heterodyne wireless optical communication systems over gamma-gamma atmospheric turbulence channels," IET Electron. Lett., vol. 44, no. 5, pp. 372-373, Feb. 2008.

[23] M. K. Simon and M.-S. Alouini, Digital Communication Over Fading Channels, 2nd ed. New York: Wiley, 2005.

Nestor D. Chatzidiamantis (S'08) was born in Los Angeles, CA, in 1981. $\mathrm{He}$ received the Diploma degree in electrical and computer engineering from the Aristotle University of Thessaloniki, Thessaloniki, Greece, and the M.Sc. degree in telecommunication networks and software from the University of Surrey, Guildford, U.K. in 2005 and 2006, respectively. Since November 2007, he has been working toward the Ph.D. degree at the Aristotle University of Thessaloniki.

His research interests include performance analysis of wireless communication systems over fading channels, communications theory, and free-space optical communications.
Harilaos G. Sandalidis was born in Florina, Greece, in 1972. He received the Diploma degree in electronics and computer engineering and the M.Sc. degree in business administration at the Production Engineering and Management Department, Technical University of Crete, Chania, Greece, in 1995 and 1998, respectively, and the M.Sc. degree in radio frequency and microwave communications and the Ph.D. degree in telecommunications in the Electronics and Telecommunications (former Electronics and Electrical Engineering) Department, University of Bradford, Bradford, U.K., in 1996 and 2002, respectively.

Between 1996 and 2001, he was a Research Assistant at the Telecommunications Systems Institute of Crete. In 2002, he joined TEMAGON (former OTE Consulting). He was also a Senior Investigator for the Greek Ombudsman. In March 2009, he joined the University of Central Greece, where he is currently a Lecturer in the Department of Computer Science and Biomedical Informatics. His major research interests include optical wireless communications, computational intelligence, and heuristic optimization techniques regarding their application to the telecommunications field.

George K. Karagiannidis (M'97-SM'04) was born in Pithagorion, Samos Island, Greece. He received the University Diploma (5 years) and Ph.D. degree, both in electrical and computer engineering, from the University of Patras, $\mathrm{Pa}-$ tras, Greece, in 1987 and 1999, respectively.

From 2000 to 2004, he was a Senior Researcher at the Institute for Space Applications and Remote Sensing, National Observatory of Athens, Greece. In June 2004, he joined Aristotle University of Thessaloniki, Thessaloniki, Greece, where he is currently an Associate Professor of digital communications systems in the Electrical and Computer Engineering Department and Head of the Telecommunications Systems and Networks Laboratory. His current research interests include digital communications systems with emphasis on cooperative communication, adaptive modulation, multiple-input multiple-output systems, optical wireless, and underwater communications. He is the author or coauthor of more than 120 technical papers published in scientific journals and presented at international conferences. He is also a coauthor of three chapters in books and author of the Greek edition book on Telecommunications Systems.

Dr. Karagiannidis is a member of Technical Program Committees for several IEEE conferences as International Conference on Communications, IEEE Global Telecommunications Conference, etc. He is a member of the editorial board of the IEEE TRANSACTIONS ON COMMUNICATIONS, a Senior Editor of the IEEE COMMUNICATIONS LETTERS and a Lead Guest Editor of the special issue on "Optical Wireless Communications" of the IEEE JouRnaL ON SELECTED AREAS ON COMMUNICATIONS. He is corecipient of the Best Paper Award of the Wireless Communications Symposium in IEEE International Conference on Communications, Glasgow, U.K., June 2007. He is the Chair of the IEEE Communications Society Greek Chapter.

Michail Matthaiou (S'05-M'08) was born in Thessaloniki, Greece, in 1981 He received the Diploma degree (5 years) in electrical and computer engineering from the Aristotle University of Thessaloniki, Thessaloniki, Greece, in 2004, the M.Sc. (with distinction) degree in communication systems and signal processing from the University of Bristol, Bristol, U.K., in 2005, and Ph.D. degree from the University of Edinburgh, U.K., in 2008.

From September 2008 to May 2010, he was a Postdoctoral Research Associate at the Institute for Circuit Theory and Signal Processing, Munich University of Technology, Munich, Germany. In June 2010, he joined Chalmers University of Technology, Gothenburg, Sweden, where he is currently an Assistant Professor. His research interests include signal processing for wireless communications, random matrix theory and multivariate statistics for multiple-input multiple-output systems, and performance analysis of fading channels.

Dr. Matthaiou is a corecipient of the 2006 IEEE Communications Chapter Project Prize for the best M.Sc. dissertation in the area of communications. 